\title{
The role of pharmacokinetic modelling and computer simulation in drug formulation design
}

\author{
I. Grabnar ${ }^{\mathrm{a}, *}$, A. Belič ${ }^{\mathrm{b}}$, A. Mrhar ${ }^{\mathrm{a}}$, R. Karba ${ }^{\mathrm{b}}$ \\ ${ }^{a}$ Faculty of Pharmacy, Askerčeva 7, University of Ljubljana, 1000 Ljubljana, Slovenia \\ ${ }^{\mathrm{b}}$ Faculty of Electrical Engineering, Trźaška 25, University of Ljubljana, 1000 Ljubljana, Slovenia
}

Received 8 December 1997

\begin{abstract}
The purpose of this study is to develop a formulation for a once-a-day peroral application with controlled release of nitrendipine. The second aim of the study is to define by the use of modelling and computer simulation those critical points in the development of the formulation, the knowledge and controlling of which brings a rationalisation in the sense of time and material. (C) 1998 Elsevier Science Ltd. All rights reserved.
\end{abstract}

Keywords: Nitrendipine; Pharmacokinetics; Modelling and simulation; Drug formulation design; Optimisation of antihypertensive effect

\section{Introduction}

The design of drug formulations is based on the principles of pharmacokinetics, biopharmaceutics and pharmaceutical technology. In accordance with the updated trends, formulations that reduce to the minimum the possibility of undesired toxic effects and increase to the maximum the possibility of desired therapeutic effects should be developed. Therefore a methodology for drug formulations design is needed that would in the final phase ensure the achievement of the set goal in the best possible way. The framework of the methodology is a step-wise iterative procedure that includes the data on pharmacokinetic and biopharmaceutical properties of the drug itself, the data on the therapeutic range of plasma concentrations, the analytical method for the identification of drug concentrations in artificial and biological fluids, the procedures of mathematical modelling and computer simulation, in vitro and in vivo

\footnotetext{
* Corresponding author. Tel.: +386-61-1769-500; Fax: +386-61-1258-031; E-mail: iztok.grabnar@ffa.uni-lj.si.
} 
testing procedures and a clearly defined purpose of the study. The result of a step-wise iterative procedure is an instruction that contains data on the mode of application and on the formulation with a controlled release of the drug [1].

\section{Starting points for the development of the nitrendipine pharmacokinetic model}

Nitrendipine [2] produces antihypertensive effect through blockade of the calcium conducting channels. After peroral application it is rapidly and completely absorbed in portal blood, while in the liver it is subjected to extensive presystemic metabolism the consequence of which is approximately $25 \%$ absorption into the systemic blood circulation. The absolute bioavailability does not depend on the size of the administered doze and on its release rate, what is manifested in linearity of kinetics. It has been proved, that no saturation of the liver enzymes occurs with the doses of 5 to $40 \mathrm{mg}$. The biological half life of nitrendipine is about $12 \mathrm{~h}$ (range 2-24 h). Besides biological variability, different sensitivity of analytical methods as a major cause is reflected in the great span of this value. Till 1985 (the period of first clinical trials) HPLC analytics was used and its sensitivity is $1-2 \mu \mathrm{g} / \mathrm{l}$. In the year 1987 the capillary GC-MS analytics was developed and essentially improved the sensitivity up to the range $0,02-$ $0,1 \mu \mathrm{g} / \mathrm{l}$. The authors using HPLC analytics reported on biological half life of $3.5 \mathrm{~h}$ in an average from 2 to $5 \mathrm{~h}$, while the authors using GC assay determined the value of $10 \mathrm{~h}$ in the average (from 9 to $11 \mathrm{~h}$ ). The influence of the analytical method is also evident in the results of the pharmacokinetic analysis. The HPLC methodology permitted the identification of two phases, while GC analytics drew the attention to the existence of the third phase in the plasma concentration profile. This finding has a key meaning in understanding the relation between concentration and effect. Namely, in the literature there could not be found any data that would combine the plasma concentration of nitrendipine with the antihypertensive effect. These investigations resulted in the presumption that there exists a correlation between the antihypertensive action and the nitrendipine concentration in the calcium channels. One can hypothesise that there exists the 'deep' peripheral compartment (calcium channels?) where the drug accumulates, maintains its pharmacodynamic effect and returns very slowly back to the central compartment. The latter may be the reason for the observed low plasma concentrations in the terminal phase. Moreover, essential cumulation of nitrendipine in the 'deep' compartment and considerable oscillations of drug levels in that compartment can be expected irrespective of the release rate of nitrendipine from formulation administered perorally.

\section{Methods}

The data base for this study, was taken from the reference study [3] in which the authors investigated the pharmacokinetics of nitrendipine following the application in different doses in an intravenous injection (formulation A) and perorally in a gelatinous capsule containing solution (formulation B) as well as in a tablet with instant release (formulation C). Nitrendipine plasma concentrations were determined by HPLC method. Only the plasma concentration profile obtained following an intravenous injection of $2 \mathrm{mg}$ of nitrendipine was 
pharmacokinetically analysed with the method of stripping. Their statement that a twocompartment model (with two phases in the concentration profile) represents a satisfactory approximation of nitrendipine pharmacokinetics, served us as a starting point for a gradual development of a model in accordance with the purposes of modelling. As a tool for developing a model, compartmental pharmacokinetic modelling [4] and digital simulation package Simulink $[5,6]$ were chosen.

\section{Results and discussion}

In Fig. 1 a model is shown that can be used multipurposely with respect to different modes of administration and different interpretations of the distribution of nitrendipine in the body.

The model that contains the compartments $C_{\mathrm{P}}, U_{\mathrm{d} 1}$ and $U_{\mathrm{e} 2}$ illustrates the starting situation (intravenous application in the form of an injection, a two-compartment model with the central compartment and one peripheral compartment, systemic metabolism). The results of simulation are shown in Fig. 2.

As the last measurement at time $8 \mathrm{~h}$ is already on the limit of detection with the HPLC method the authors could not experimentally determine the course of plasma concentrations in the time interval between the 8th and 24th hour. So, there exists the possibility that the course foreseen with the two-compartment model may also be different. In regard to the suggestion

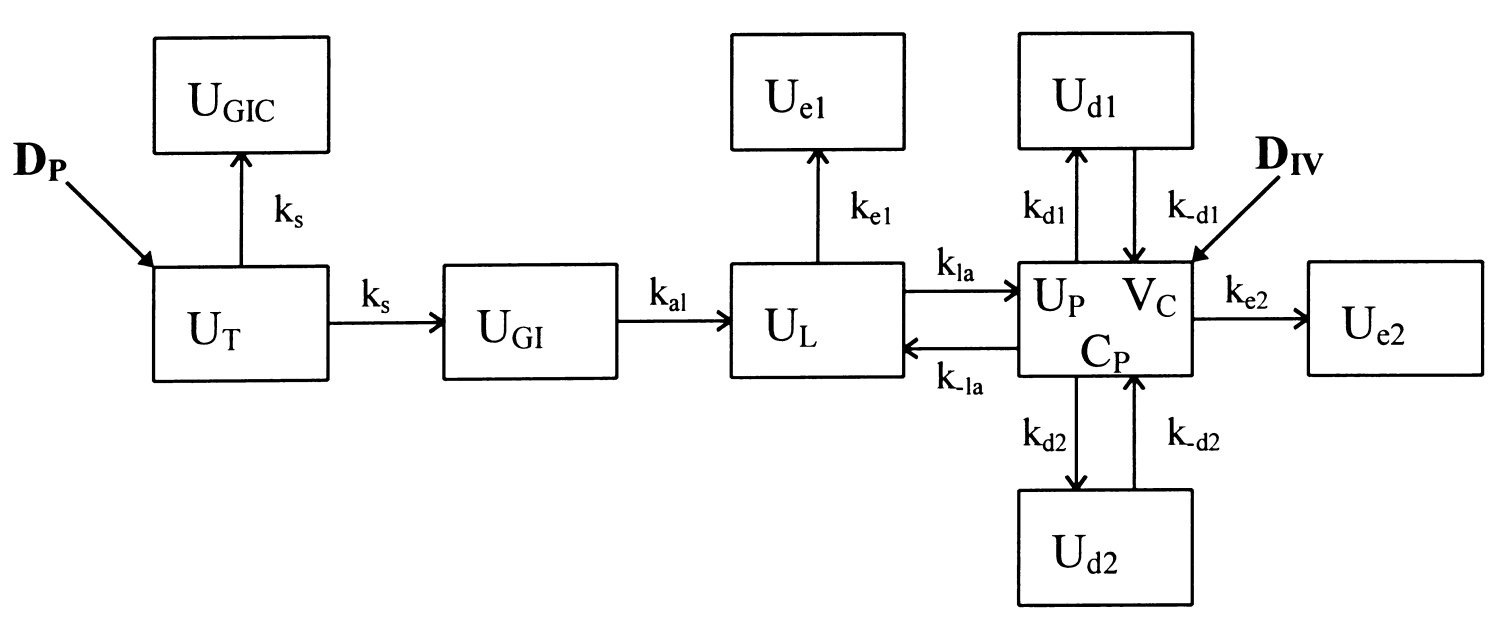

Fig. 1. Compartmental pharmacokinetic model of nitrendipine. $U_{\mathrm{T}}$ levels in the tablet, $U_{\mathrm{GI}}$ levels in the gastrointestinal tract, $U_{\mathrm{GIC}}$ cumulative levels in the gastrointestinal tract, $U_{\mathrm{L}}$ levels in the liver, $U_{\mathrm{P}}$ levels in the plasma (the central compartment), $C_{\mathrm{P}}$ plasma concentrations, $V_{\mathrm{C}}$ volume of distribution of the central compartment, $U_{\mathrm{d} 1}$ levels in the 'shallow' peripheral compartment, $U_{\mathrm{d} 2}$ levels in the 'deep' peripheral compartment, $U_{\mathrm{e} 2}$ levels of metabolites after presystemic metabolism, $U_{\mathrm{PO}}$ levels of metabolites after systemic metabolism, $D_{\mathrm{PO}}$ peroral dose, $D_{\mathrm{IV}}$ intravenous dose, $k_{\mathrm{s}}$ release rate constant, $k_{\mathrm{al}}$ absorption rate constant into the portal blood circulation, $k_{\mathrm{la}}$ and $k_{-\mathrm{la}}$ absorption rate constants into the central blood circulation, $k_{\mathrm{d} 1}$ and $k_{-\mathrm{d} 1}$ distribution rate constants into the 'shallow' compartment, $k_{\mathrm{d} 2}$ and $k_{-\mathrm{d} 2}$ distribution rate constants into the 'deep' compartment, $k_{\mathrm{e} 1}$ and $k_{\mathrm{e} 2}$ metabolising rate constants. 
we wished to verify the hypothesis whether the three-compartment model eventually enables an equal quality of fit in the interval between 0th and 8th hour, while in the interval between 8th and 24th hour the concentration of nitrendipine in plasma does not decrease to a negligible value. We chose a model that contains compartments $C_{\mathrm{P}}, U_{\mathrm{d} 1}, U_{\mathrm{d} 2}$ and $U_{\mathrm{e} 2}$ (Fig. 3) to illustrate the new situation (intravenous application in the form of an injection, three-

$\mathbf{a}$

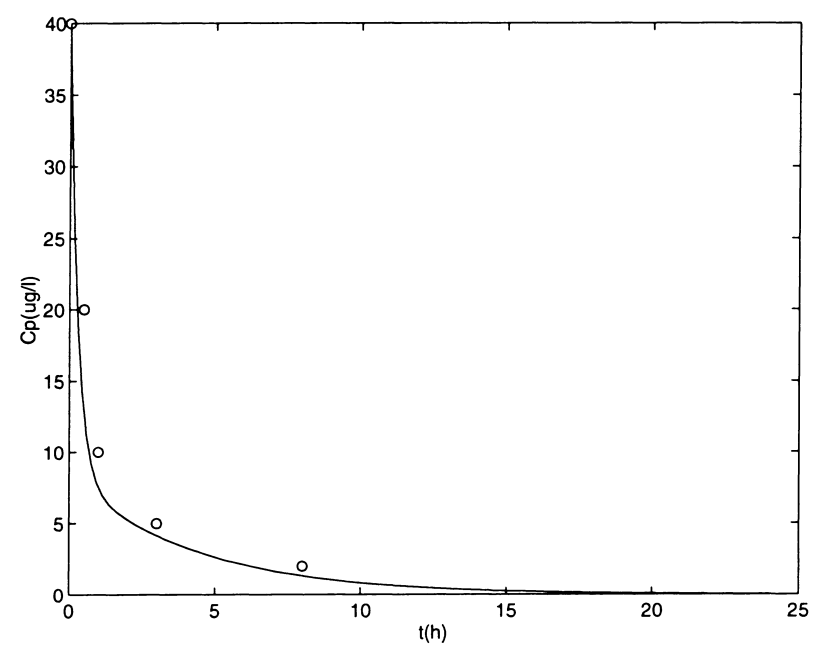

b

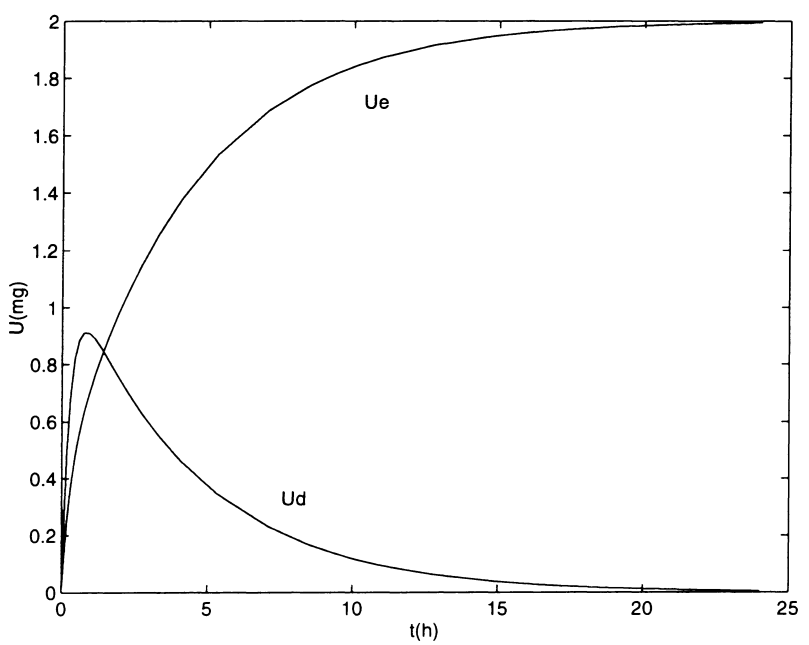

Fig. 2. Course of nitrendipine levels following the application of a $2 \mathrm{mg}$ dose in an intravenous injection (twocompartment model). (a) Concentrations in the plasma, the points - in vivo measurements, the curve - response of the model. (b) Quantities in the peripheral compartment and in the compartment of metabolites. 
compartment model with the central compartment, a 'shallow' peripheral compartment, a 'deep' peripheral compartment and systemic metabolism).

The results of simulation on Fig. 3a confirmed our hypothesis since the goodness of fit in the central compartment is comparable to that in Fig. 2a. Model response showed that in the time interval between the 8th and 24th hour the nitrendipine levels are significantly high only in the compartment $U_{\mathrm{d} 2}$. Furthermore, the course of nitrendipine levels in this compartment may

$\mathbf{a}$

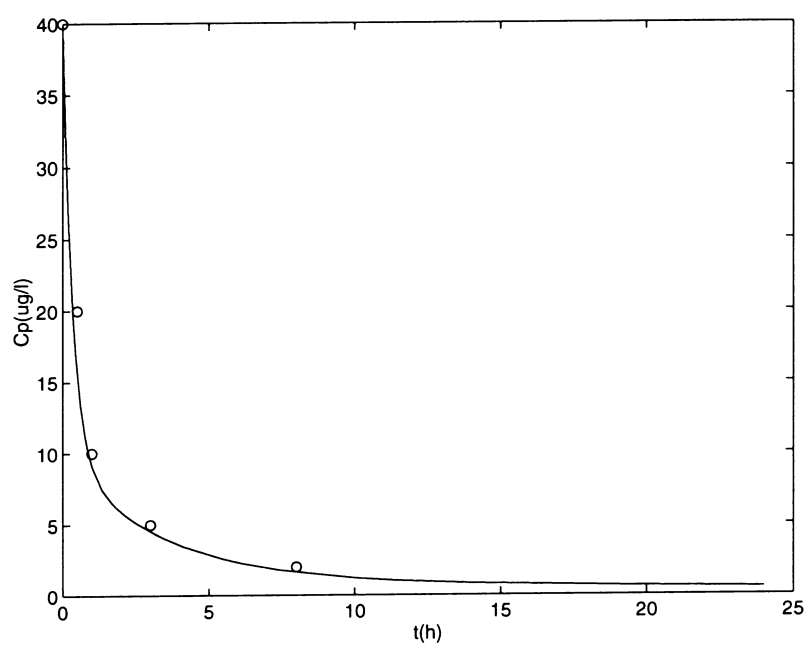

b

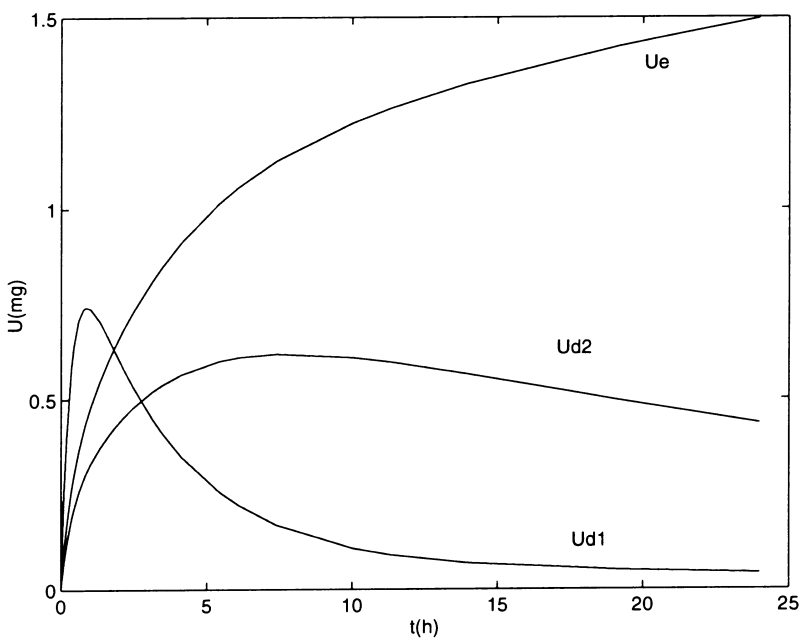

Fig. 3. Course of nitrendipine levels following the application of a $2 \mathrm{mg}$ dose in an intravenous injection (threecompartment model). (a) Concentrations in the plasma, the points - in vivo measurements, the curve - response of the model. (b) Quantities in the peripheral compartments and in the compartment of metabolites. 
support the statement that the antihypertensive effect of nitrendipine correlates better with the concentrations on the site of action (calcium channels) than with plasma concentrations, if we suppose that calcium channels are located in the $U_{\mathrm{d} 2}$ compartment.

As the purpose of our investigation was to develop a peroral formulation with a controlled release of nitrendipine, we continuing our investigation extended the three-compartment model

a

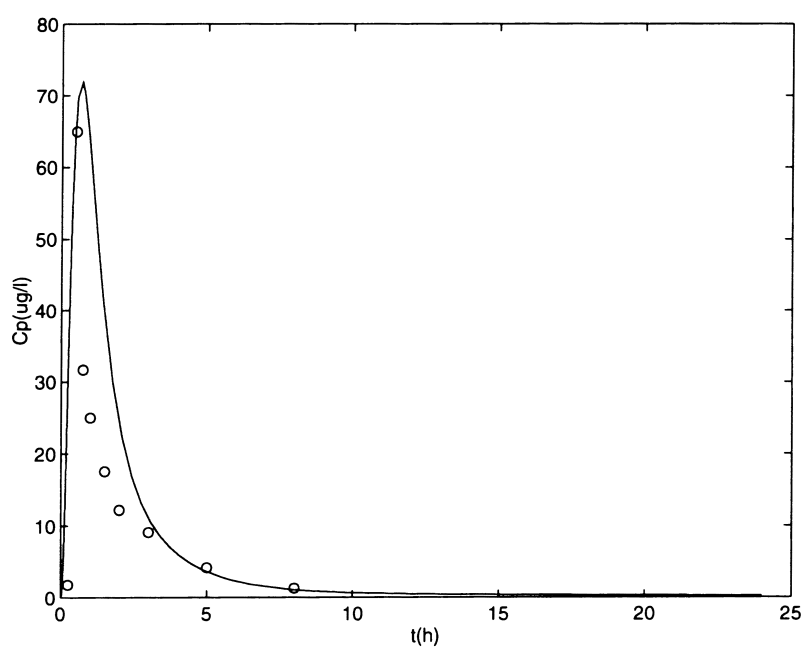

b

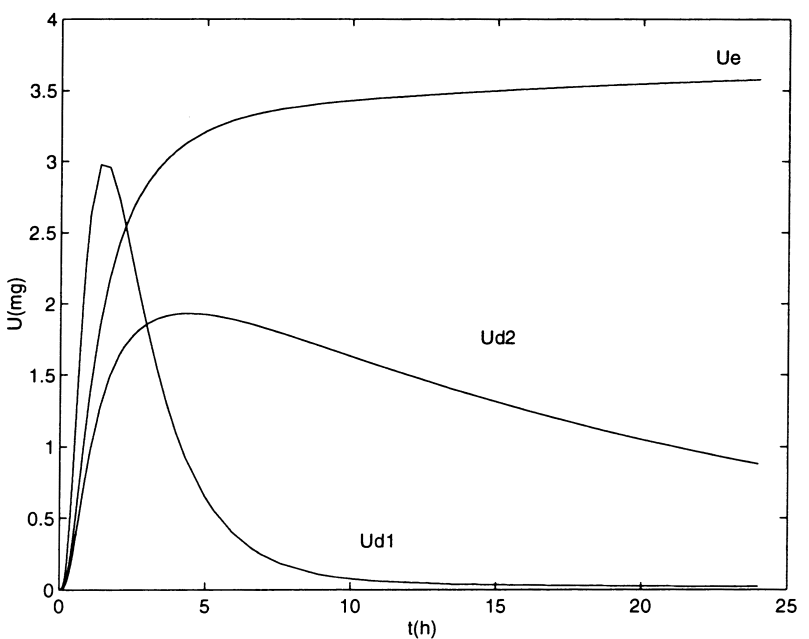

Fig. 4. Course of nitrendipine levels following the application of a $30 \mathrm{mg}$ dose in a peroral gelatinous capsule containing solution (three-compartment model). (a) Concentrations in the plasma, the points - in vivo measurements, the curve - response of the model. (b) Quantities in the peripheral compartments and in the compartment of metabolites. 
with the compartments that enable the simulation of peroral application (the compartments $U_{\mathrm{T}}, U_{\mathrm{GI}}, U_{\mathrm{GIC}}$ ) and the simulation of presystemic metabolism in the liver (the compartments $U_{\mathrm{L}}$ and $\left.U_{\mathrm{e} 1}\right)$. Two different gastrointestinal compartments have been included in the model: a cumulative one, which gives information similar to the in vitro dissolution profile of the drug and the actual one, which simulates the levels comparable to those present in vivo. The results of investigations concerning the influence of drug formulation, i.e. the release rate on the

a

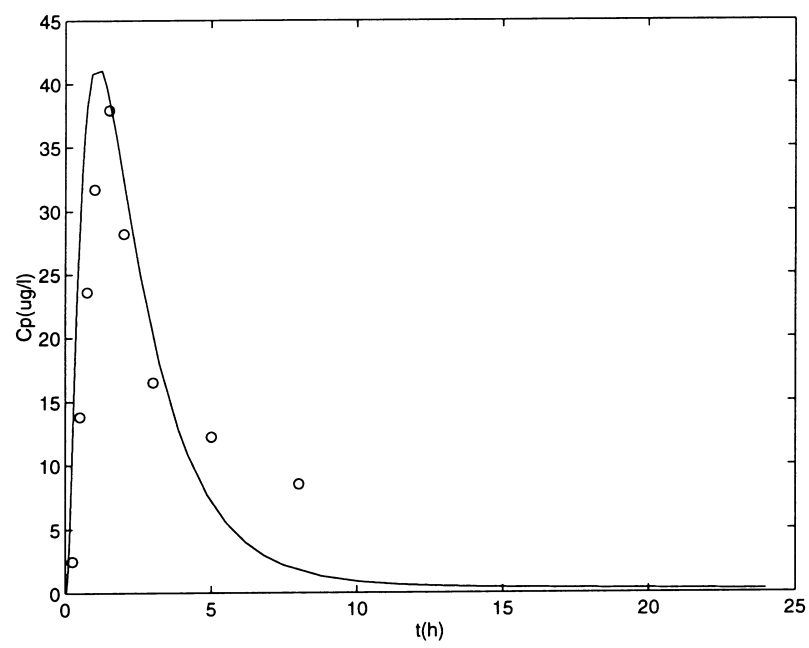

b

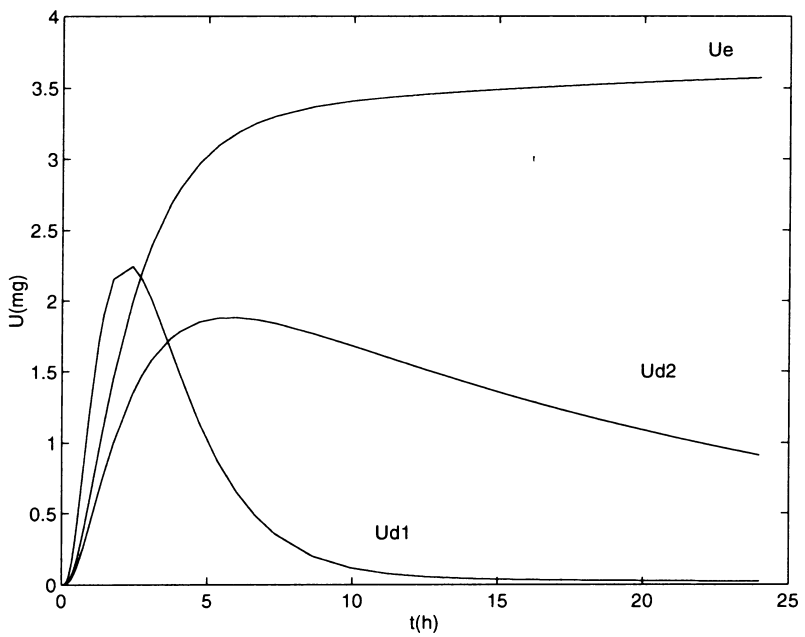

Fig. 5. Course of nitrendipine levels following the application of a $30 \mathrm{mg}$ dose in a peroral tablet with instant release (three-compartment model). (a) Concentrations in the plasma, the points - in vivo measurements, the curve - response of the model. (b) Quantities in the peripheral compartments and in the compartment of metabolites. 
nitrendipine levels in all the compartments of the model in Fig. 1 following the administration of gelatinous capsule (formulation B) and tablet with instant release, with $30 \mathrm{mg}$ nitrendipine each are shown in the Figs. 4 and 5.

Table 1 summarises the values of identified nitrendipine pharmacokinetic parameters. As seen from Figs. 4 and 5 and Table 1, optimal accordance between the model response and the experimental data for both peroral formulations was reached only by modulating the parameter $k_{\mathrm{s}}$, what indicates that release is the process that exerts a deciding influence on the pharmacokinetics of the drug and herewith also on its pharmacological effect. The influence of the release rate is to a high degree manifested in the plasma concentration profile. More interesting results are shown when analysing the profiles in $U_{\mathrm{d} 2}$. In this case the influence of the release rate is manifested in essentially smaller degree. The levels in $U_{\mathrm{d} 2}$ are comparable at $24 \mathrm{~h}$ and they range about $1 \mathrm{mg}$ regardless of the release rate. As well as the reason for the change of the plasma concentration profile for the formulations $\mathrm{B}$ and $\mathrm{C}$, which was identified uniquely, also behaviour after multiple dosing, i.e. in cases when chronic therapy is indicated can be predicted. It is known that a change in release rate influences only the extent of oscillation of drug levels and not the average steady state values of drug levels in those compartments where cumulation occurs. This is also confirmed by the results of simulation of multiple dosing of a $20 \mathrm{mg}$ nitrendipine dose in formulations B and C, shown in Fig. 6.

It is obvious from Fig. 6a that nitrendipine does not cumulate in plasma (central compartment) after multiple dosing. Furthermore, the oscillations of nitrendipine concentrations are considerable and the average values differ significantly. To this statement it can be added that nitrendipne also does not cumulate in the 'shallow' compartment, while the cumulation is essential in the 'deep' compartment irrespective of the release rate from the

Table 1

Parameters of the nitrendipine pharmacokinetic model calculated from the concentration course in the plasma (central compartment) following the application of nitrendipine in intravenous injection (formulation A), in gelatinous capsule (formulation B) and in tablet with instant release (formulation C). (2-two-compartment model, 3-threecompartment model)

\begin{tabular}{lllcc}
\hline & \multicolumn{3}{l}{ Formulation } & \\
\cline { 2 - 5 } Parameter $\left[\mathrm{h}^{-1}\right]$ & $\mathrm{A} 2$ & $\mathrm{~A} 3$ & $\mathrm{~B} 3$ & $\mathrm{C} 3$ \\
\hline$k_{\mathrm{d} 1}$ & 1.94 & 1.40 & 1.40 & 1.40 \\
$k_{-\mathrm{d} 1}$ & 0.91 & 0.94 & 0.94 & 0.94 \\
$k_{\mathrm{d} 2}$ & & 0.37 & 0.37 & 0.37 \\
$k_{-\mathrm{d} 2}$ & & 0.05 & 0.05 & 0.05 \\
$k_{\mathrm{e} 2}$ & 0.89 & 0.52 & 0.52 & 0.52 \\
$K_{\mathrm{e} 1}$ & & & 1.45 & 1.45 \\
$k_{\mathrm{la}}$ & & & 3.70 & 3.70 \\
$k_{-\mathrm{la}}$ & & & 8.80 & 8.80 \\
$k_{\mathrm{a} 1}$ & & & 10.0 & 10.0 \\
$k_{\mathrm{S}}$ & & & 3.10 & 0.90 \\
\hline
\end{tabular}


a

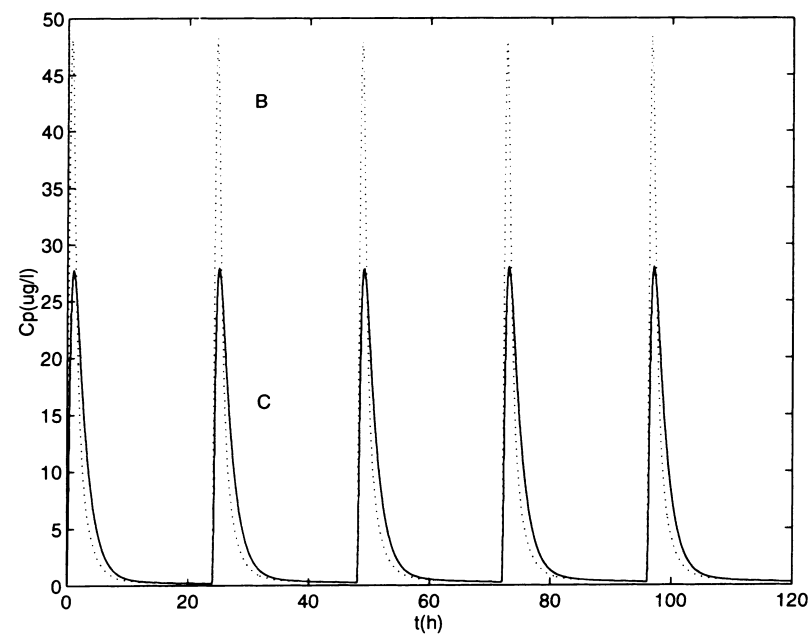

$\mathbf{b}$

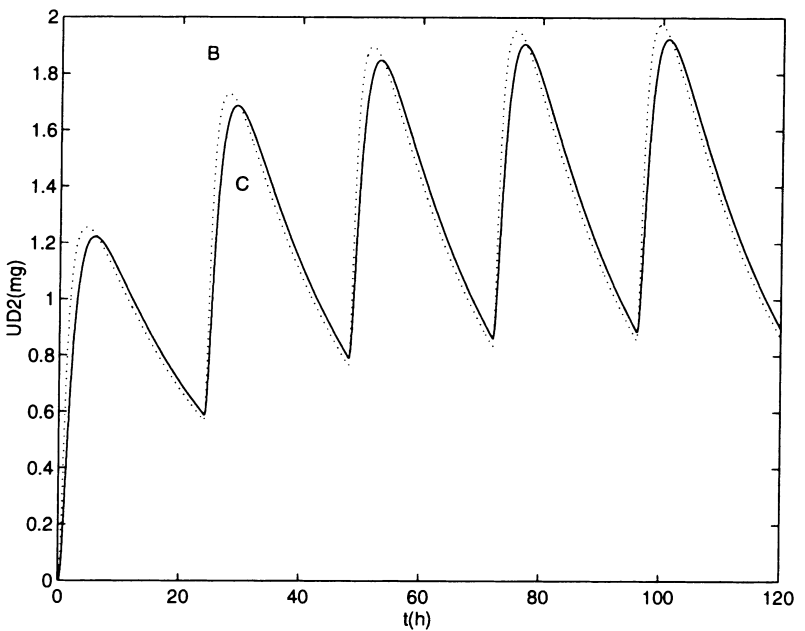

Fig. 6. Simulated course of the nitrendipine levels after multiple dosing of formulation B (dotted line) and C (solid line), $24 \mathrm{~h}$ interval, three-compartment model. (a) Plasma concentrations, (b) levels in the 'deep' compartment.

formulation administered perorally. In Fig. $6 \mathrm{~b}$ the profiles in $U_{\mathrm{d} 2}$ after multiple dosing are compared with respect to the difference in the release rate. The average level in the steady state is equal (1.25 mg for a $20 \mathrm{mg}$ dose), while the oscillations are the function of the release rate. It can be seen that the difference in oscillations is much smaller than the one observed in plasma. The conclusion drawn from the results of simulation of multipleperoral dose regimen are supported by findings of Johnson et al. [7]. He demonstrated that blood pressure in patients with essential hypertension continued to decline over a $6 \mathrm{~h}$ period after peroral dosing 
as the plasma concentration of nitrendipine decreased. By the aid of supposition that calcium channels are located in the 'deep' peripheral compartment we provide the explanation that essential prolongation of pharmacodynamic effect is a result of high nitrendipine levels in the 'deep' peripheral compartment, i.e. calcium channels during the period of $24 \mathrm{~h}$. In the case of plasma concentration profile this period amounts to only $8 \mathrm{~h}$.

Overall, the results of simulation permitted us the following conclusions: there is justified a development of a formulation for peroral application releasing nitrendipine with first order rate constant ranging between 0.5 and $1.5 \mathrm{~h}^{-1}$, with respect to low solubility of nitrendipine it is essential to prepare a formulation which would release at least $90 \%$ of the dose in $10 \mathrm{~h}$ at the very latest and there is justified the once-a-day application of nitrendipine in the formulation with controlled release in the case of chronic therapy.

\section{Summary}

The purpose of this contribution is to present the role of pharmacokinetic modelling and computer simulation in the design of controlled release formulations and to define those critical points in the procedure, the knowledge and controlling of which brings a rationalisation in the timely and material sense. The approach is illustrated by the design of peroral formulation with controlled release of nitrendipine, an antihypertensive drug from dihydropyridine series.

Nitrendipine is rapidly and completely absorbed in portal blood after peroral application, while in the liver it is subjected to extensive presystemic metabolism, the consequence of which is approximately $25 \%$ absorption to systemic blood circulation. The absolute bioavailability does not depend on the size of the administered dose and on its release rate. When analysing plasma concentration profiles by the method of residuals, the HPLC methodology permitted the identification of two phases, while GC/MS with improved sensitivity drew the attention to the existence of the third phase in the plasma concentration profile resulting in the significant prolongation of biological half life. This finding had a key meaning in understanding the relationship between concentration and effect, and represented a critical point in the process of nitrendipine formulation design. Namely, in the literature there could not be found any data that would combine the plasma concentration of nitrendipine with antihypertensive effects. This investigation resulted in the presumption that there exists a correlation between the antihypertensive action and the nitrendipine concentration in the calcium channels. We hypothesised that there exists a 'deep' compartment (calcium channels?) where the drug accumulates, maintains its pharmacodynamic effect and returns very slowly back to the central compartment. The latter may be the reason for the observed low plasma concentrations in the terminal phase. Moreover, we expected essential cumulation of nitrendipine in the 'deep' compartment and considerable oscillations of drug levels in the compartment, irrespective of the release rate of nitrendipine from formulation administered perorally. For the development and application of nitrendipine pharmacokinetic study, the data base was taken from the reference study, in which authors investigated the pharmacokinetics of nitrendipine following the application of different doses in different dosage forms. Pharmacokinetic compartmental analysis based on digital simulation package Simulink was carried out with the use of the 
composed two-(central and peripheral) and three-(central, shallow and deep) compartment model. The results of simulation in the case of single-dose regimen confirmed our hypothesis regarding the structure of the model, since the goodness of fit in central compartment (plasma) in the case of three compartment model was excellent and antihypertensive effect correlated better with the course of nitrendipine levels in the deep compartment. This finding led us to the supposition that calcium channels are located in the deep compartment. Consequently, the release of nitrendipine from peroral formulation and the dosage interval must be adjusted to provide optimal profile in this compartment. The results of simulation in the case of multiple dose regimen, confirming our hypothesis regarding cumulation and oscillations in the deep compartment, permitted us the following conclusions: there is justified a development of a formulation for peroral application releasing nitrendipine with first order rate constant ranging between 0.5 and $1.5 \mathrm{~h}^{-1}$, with respect to low solubility of nitrendipine it is essential to prepare a formulation which would release at least $90 \%$ of the dose in $10 \mathrm{~h}$ at the very latest and there is justified the once-a-day application of nitrendipine in the formulation with controlled release in the case of chronic therapy.

\section{References}

[1] A. Kristl, D. Vojnovic, R. Karba, A. Mrhar, F. Rubessa, B. Zupančič, Mixture design of theophyline retard formulation, International Journal of Pharmaceutics 99 (1993) 33.

[2] K.L. Goa, E.M. Sarkin, Nitrendipine, a review of its pharmacodynamic and pharmacokinetic properties and therapeutic efficacy in the treatment of hypertension, Drugs 33 (1987) 123.

[3] K.D. Raemsch, J. Sommer, Pharmacokinetics and metabolism of nitrendipine, in: Scriabne et al. (Eds.), Nitrendipine, Urban and Schwarzenberg, 1984, p. 409.

[4] C. Remunan, A. Mrhar, S. Primoźič, R. Karba, J.L. Vila-Jato, Sustained release nifedipine formulations: moment, modelling and simulation as pharmacokinetic analysis approach, Drug Development Industrial Pharmacy 18 (1992) 187.

[5] Matlab Reference Guide, The MathWorks, 1992.

[6] R. Karba, A. Mrhar, B. Zupančič, M. Atanasijević-Kunc, A. Belič, The role of simulation tool in histamine pharmacokinetical model development, in: T.I. Oren, L.G. Birta (Eds.), Proceedings of the 1995 summer computer simulation conference, Ottawa, 1995, p. 685.

[7] B.F. Johnson, L. Romero, R. Mrwaha, Hemodynamic and metabolic effects of the calcium channel blocking agent nitrendipine, Clinical Pharmacology and Therapeutics 39 (1986) 389.

Iztok Grabnar received his B.Sc. in 1995 from the Faculty of Pharmacy, University of Ljubljana, Slovenia. Currently he is a probationary assistant at the Faculty of Pharmacy, University of Ljubljana, Slovenia. The main fields of his research are modelling and simulation in pharmacokinetics and pharmacodynamics and pharmaceutical informatics.

Aleš Belič was born in 1971. He received his B.Sc. degree from Faculty of Electrical Engineering, University of Ljubljana, Slovenia in 1994. In 1996 he was awarded the Prešeren's award for the students. He is currently a probationary assistant at the Faculty of Electrical Engineering, University of Ljubljana, Slovenia. The main field of his research is modelling and simulation in pharmacokinetics, pharmacodynamics and related sciences.

Aleš Mrhar received his B.Sc. (1975), M.Sc. (1978) and Ph.D. (1984) degrees from the Faculty of Pharmacy, University of Ljubljana, Slovenia. His research interests include modelling and simulation in pharmacokinetics, biopharmaceutics and pharmaceutical technology, design, preparation and evaluation of drug delivery systems as well as pharmaceutical informatics. Currently he is a Professor at the Faculty of Pharmacy, University of Ljubljana, Slovenia. 
Rihard Karba received his B.Sc. (1972), M.Sc. (1975) and Ph.D. (1981) degrees from the Faculty of Electrical Engineering, University of Ljubljana, Slovenia. His research interests include modelling and simulation of dynamic systems, as well as modelling and simulation in pharmacokinetics and biomedicine. He is currently a Professor at the Faculty of Electrical Engineering, University of Ljubljana, Slovenia. 